\title{
Orbital migration and mass-semimajor axis distributions of extrasolar planets
}

\author{
Shigeru Ida ${ }^{1}$ and D. N. C. $\operatorname{Lin}^{2,3}$ \\ 1 Tokyo Institute of Technology, Ookayama, Meguro-ku, Tokyo 152-8551, Japan \\ email: ida@geo.titech.ac.jp \\ ${ }^{2}$ UCO/Lick Observatory, University of California, Santa Cruz, CA 95064, USA; Kavli Institute \\ of Astronomy \& Astrophysics, Peking University, Beijing, China \\ email: lin@ucolick.org
}

\begin{abstract}
Here we discuss the effects of type-I migration of protoplanetary embryos on mass and semimajor axis distributions of extrasolar planets. We summarize the results of Ida \& Lin (2008a, 2008b), in which Monte Carlo simulations with a deterministic planet-formation model were carried out. The strength of type-I migration regulates the distribution of extrasolar gas giant planets as well as terrestrial planets. To be consistent with the existing observational data of extrasolar gas giants, the type-I migration speed has to be an order of magnitude slower than that given by the linear theory. The introduction of type-I migration inhibits in situ formation of gas giants in habitable zones (HZs) and reduces the probability of passage of gas giants through HZs, both of which facilitate retention of terrestrial planets in HZs. We also point out that the effect of magneto-rotational instability (MRI) could lead to trapping of migrating protoplanetary embryos in the regions near an ice line in the disk and it significantly enhances formation/retention probability of gas giants against type-I migration.
\end{abstract}

Keywords. planetary systems: formation, solar system: formation, stars: statics

\section{Introduction}

\subsection{Difficulty of too rapid type-I migration}

In the core accretion scenario for planet formation (e.g., Hayashi et al. 1985), migration of planetary embryos due to tidal interactions of embedded embryos with surrounding disk gas (e.g., Goldreich \& Tremaine 1980, Ward 1986) is one of the most serious problems. Analytic studies suggest that isolated embryos lose angular momentum to the disk exterior to their orbits faster than that they gain from the disk interior to their orbits. This torque imbalance leads to "type-I" migration.

The migration timescales for a planet with mass $M_{p}$ and orbital radius $r$ (orbital frequency $\left.\Omega_{\mathrm{K}}\right)$ in a disk with gas surface density $\Sigma_{g}\left(\propto r^{-p}\right)$ and sound velocity $c_{s}$ around a host star with mass $M_{*}$ is given by (Tanaka et al. 2002)

$$
\begin{aligned}
\tau_{\mathrm{mig} 1} & =\frac{r}{\dot{r}} \simeq \frac{1}{2.728+1.082 p}\left(\frac{c_{s}}{a \Omega_{\mathrm{K}}}\right)^{2} \frac{M_{*}}{M_{p}} \frac{M_{*}}{a^{2} \Sigma_{g}} \Omega_{\mathrm{K}}^{-1} \\
& \simeq 7 \times 10^{4} \times\left(\frac{\Sigma_{g}}{\Sigma_{g, \mathrm{MMSN}}}\right)^{-1}\left(\frac{M_{p}}{M_{\oplus}}\right)^{-1}\left(\frac{r}{1 \mathrm{AU}}\right)^{p}\left(\frac{M_{*}}{M_{\odot}}\right)^{3 / 2} \mathrm{yrs},
\end{aligned}
$$

where $\Sigma_{g, \mathrm{MMSN}}=1700(\mathrm{r} / 10 \mathrm{AU})^{-3 / 2} \mathrm{gcm}^{-2}$ is $\Sigma_{g}$ of the minimum-mass solar nebula (MMSN) model (Hayashi 1981). For an Earth-mass planet at 1AU and a 10 Earthmass core at $\sim 5 \mathrm{AU}, \tau_{\mathrm{mig} 1} \sim 10^{5}$ years in a disk similar to MMSN, which is much shorter than the observationally inferred lifetime of protoplanetary disks $\left(\sim 10^{6}-10^{7}\right.$ years $)$. Since more than several $M_{\oplus}$ may be required for a core to start runaway gas accretion onto the 
core (see eq. [1.2]), the short migration timescale implies the difficulty in formation of gas giants. However, it is apparently inconsistent with observationally postulated probability ( $\gtrsim 10 \%$ ) of solar type stars harboring gas giants (e.g., Marcy et al. 2005, Mayor et al. 2005). Furthermore, the mostly refractory compositions of the terrestrial planets in the present-day Solar system suggest that they probably did not migrate to their present locations from regions well beyond the ice line.

\subsection{Preservation of solid materials in terrestrial planet regions}

The difficulty for formation of terrestrial planets is less serious if oligarchic growth model (Kokubo \& Ida 1998, Kokubo \& Ida 2002) is considered. The oligarchic growth model suggests that growth of planetary embryos is stalled at their isolation mass that is about a Mars mass in the terrestrial planet regions in the disk similar to MMSN, until $\Sigma_{g}$ is depleted to $10^{-3}$ times of that of MMSN and coagulation between the embryos start (Kominami \& Ida 2002). It prevents rapid depletion of solid materials in full amount of disk gas, since the migration timescale of embryos is $\tau_{\text {mig } 1} \sim 10^{6}$ years (eq. [1.1]), which is not significantly shorter than lifetime of protoplanetary disks. With slight reduction of type-I migration speed or slightly enhanced dust to gas ratio of the disk, enough amount of materials for the formation of Earth-mass planets can be retained (McNeil et al. 2005, Daisaka, Tanaka \& Ida 2006).

Through Monte Carlo simulations on the basis of a comprehensive treatment of the sequential planet formation scenario, Ida \& Lin (2008a) found that although uninhibited type-I migration leads to efficient self-clearing of embryos, materials of a few times $M_{\oplus}$ remain at $a \gtrsim 1 \mathrm{AU}$, almost independent of initial surface density of planetesimals $\left(\Sigma_{d}\right)$ and migration strength. At $a \gtrsim 1 \mathrm{AU}$, embryos start type-I migration well before they attain isolation mass, so after they have migrated, new-generation embryos continue to form from residual planetesimals. This repeated generation and migration slow down when $\Sigma_{d}$ declines to values comparable to or smaller than that of the MMSN model and type-I migration is no longer an effective disruption mechanism for Mars-mass embryos. With this reservoir, there is an adequate inventory of residual embryos to subsequently assemble into Earth-mass rocky planets.

\subsection{Formation and retention of cores for giant planets}

For a core to start runaway gas accretion and form a gas giant within the disk lifetime ( 10 $10^{6}-10^{7}$ years), a core of at least several $M_{\oplus}$ must accrete, because the KelvinHelmholtz contraction timescale $\left(\tau_{\mathrm{KH}}\right)$ of the gas envelope is given by

$$
\tau_{\mathrm{KH}} \simeq 10^{k 1}\left(\frac{M_{\mathrm{p}}}{M_{\oplus}}\right)^{-k 2} \mathrm{yrs},
$$

where $k 1=8-10$ and $k 2=3-4$ (see Ida \& Lin 2004a, Ida \& Lin 2004b and references therein). Note, however, that more efficient cooling of the envelope by low dust contents in inflow gas and/or non-spherically symmetric structure of the envelope can reduce $\tau_{\mathrm{KH}}$. Equation (1.1) shows that for the several $M_{\oplus}$ cores to start runaway gas accretion and form gas giants, significant reduction of type-I migration speed is required.

Cores formed after the disk gas has been severely depleted may withstand the disruption by the declining type-I migration speed (Thommes \& Murray 2006), since $\tau_{\operatorname{mig} 1} \propto$ $\Sigma_{g}^{-1}$ (eq. [1.1]). Incorporating gas accretion onto cores and the effect of type-II migration, Ida \& Lin (2008a) found that cores repeatedly form and migrate inward and those formed in "late" stage can survive for slightly reduced type-I migration. But, they showed that the formation probability of gas giant planets and hence the predicted mass and semimajor axis distributions of extrasolar gas giants are sensitively determined by the strength 
of type-I migration. They suggested that the observed fraction of solar-type stars with gas giant planets can be reproduced only if the actual type-I migration timescale is an order of magnitude longer than that deduced from linear theories $(\S 2.3)$. Note that this late formation scenario is conceptually consistent with that inferred from the noble gas enrichment in Jupiter (Guillot \& Hueso 2006).

\subsection{Retardation of type-I migration}

Retardation processes for this type-I migration are explored by many authors. One possibility for the retardation is non-linear fluid dynamical effects: self-induced unstable flow (Koller et al. 2004, Li et al. 2005), non-linear radiative and hydrodynamic feedbacks (Masset et al. 2006a), and entropy gradient (Baruteau \& Masset 2008). Magnetic field can affect wave propagation (Fromang, Terquem \& Nelson 2005, Muto \& Inutsuka 2008). Random torque due to density fluctuations caused by MRI can also overcome monotonic torque for type-I migration (Laughlin, Steinacker \& Adams 2004, Nelson \& Papaloizou 2004).

Another possibility is surface density gradient of disk gas. As shown in eq. (1.1), if $p$ is negative locally, type-I migration is slowed down or even reserved there. Masset et al. (2006b) considered an inner cavity in a gas disk and showed that migrating embryos are captured near the outer edge of the cavity. Kretke \& Lin (2007) pointed out protoplanetary disks are composed of an inactive neutral "dead zone" near the mid plane, sandwiched together by partially ionized surface layers where MRI is active. Because the main agents for removing electrons from the gas are grains (e.g., Sano et al. 2000), due to a transition in the surface density of the icy dust grains across the ice line, the thickness of the active layer decreases abruptly outside the ice line, resulting in local positive surface density. Zhang, Lin \& Kretke (2008) showed that type-I migration is stalled near the ice line with the transition. Ida \& Lin (2008b) incorporated these effects into the Monte Carlo simulation and found that the mass and semimajor axis distribution of extrasolar giant planets consistent with the observed data are reproduced with much less reduction of type-I migration strength than that required in the case without this effect.

In the below, we summarize the results of Ida \& Lin (2008a) and Ida \& Lin (2008b). In section 2 , we describe the results in the disks with $\Sigma_{g}$ that has a power-law radial dependence (Ida \& Lin 2008a). In section 3, we show the cases in which $\Sigma_{g}$ has the non-uniform radial dependence due to a coupling effect of MRI and the ice line (Ida \& Lin 2008b).

\section{Disks with surface density having a power-law radial dependence}

In a series of papers (Ida \& Lin 2004a, Ida \& Lin 2004b, Ida \& Lin 2005, Ida \& Lin 2008a), a numerical scheme to simulate the anticipated mass-semimajor distribution of planets was constructed based on a comprehensive treatment of the sequential planet formation scenario. In Ida \& Lin (2004a), we presented calculations for a solar-type stars by neglecting the effect of type-I migration. With the same assumptions, we simulated the distribution for stars with a range of metallicity, $[\mathrm{Fe} / \mathrm{H}]=\log _{10}\left((\mathrm{Fe} / \mathrm{H})_{*} /(\mathrm{Fe} / \mathrm{H})_{\odot}\right)$, and mass $\left(M_{*}\right)$ in Ida \& Lin (2004b) and Ida \& Lin (2005), respectively.

The prescriptions for planetesimal accretion, gas accretion onto cores and type-II migration for gas giant planets are essentially the same in these papers. In Ida \& Lin (2008a) and Ida \& Lin (2008b), the effect of type-I migration was included. For the details of the prescription, see Ida \& Lin (2008a). 


\subsection{Disk model}

Ida \& Lin (2008a) introduced multiplicative factors $\left(f_{d}\right.$ and $\left.f_{g}\right)$ to scale surface density of gas $\left(\Sigma_{g}\right)$ and solid components $\left(\Sigma_{d}\right)$ such that

$$
\left\{\begin{array}{l}
\Sigma_{d}=\Sigma_{d, 10} 10^{[\mathrm{Fe} / \mathrm{H}]} \eta_{\mathrm{ice}} f_{d}(r / 10 \mathrm{AU})^{-3 / 2} \\
\Sigma_{g}=\Sigma_{g, 10} f_{g}(r / 10 \mathrm{AU})^{-p}
\end{array}\right.
$$

where normalization factors $\Sigma_{d, 10}=0.32 \mathrm{~g} / \mathrm{cm}^{2}$ and $\Sigma_{g, 10}=75 \mathrm{~g} / \mathrm{cm}^{2}$ correspond to 1.4 times of $\Sigma_{g}$ and $\Sigma_{d}$ at 10AU of the MMSN model, and the step function $\eta_{\text {ice }}=1$ inside the ice line and 4.2 for $r>a_{\text {ice }}$ (Hayashi 1981). We assume that dust to gas ratio is proportional to $[\mathrm{Fe} / \mathrm{H}]$ and $[\mathrm{Fe} / \mathrm{H}]$ does not affect the distribution of $\Sigma_{g}$.

Assuming an equilibrium temperature in optically thin disk regions (Hayashi 1981),

$$
T=280\left(\frac{r}{1 \mathrm{AU}}\right)^{-1 / 2}\left(\frac{L_{*}}{L_{\odot}}\right)^{1 / 4} \mathrm{~K},
$$

where $L_{*}$ and $L_{\odot}$ are the stellar and solar luminosity. The ice line is determined by this temperature distribution as

$$
a_{\text {ice }}=2.7\left(\frac{L_{*}}{L_{\odot}}\right)^{1 / 2} \mathrm{AU} .
$$

Note that $a_{\text {ice }}$ is modulated by opacity of the disk and viscous heating (Davis 2005, Garaud \& Lin 2007).

Here, we assume that planetesimals have been formed from dust grains and $\Sigma_{d}$ is identified as surface density of planetesimals. $f_{d}$ at a given location $r$ continuously decreases with time from its initial value $f_{d, 0}$ as planetesimals are accreted by embryos that in term undergo orbital decay. Note that here semimajor axis $a$ is identified as orbital radius $r$, since we neglect evolution of orbital eccentricities. For gas component, we adopt exponential decay with decay constant $\tau_{\text {dep }}$,

$$
f_{g}=f_{g, 0} \exp \left(-t / \tau_{\mathrm{dep}}\right) .
$$

\subsection{Surface density evolution due to type-I migration}

The results on the $\Sigma_{d}$ reduction due to type-I migration are shown in Figures 1. They show $\Sigma_{d}$ at $t=10^{5}, 10^{6}$, and $10^{7}$ years (dashed, dotted, and solid lines) with $f_{g, 0}=f_{d, 0}$ and $p=1.0$ : (a) $C_{1}=1, f_{g, 0}=3$, (b) $C_{1}=1, f_{g, 0}=30$, and (c) $C_{1}=0.1, f_{g, 0}=3$, where $C_{1}$ is a reduction factor for type-I migration speed $(\dot{r})$ from the linear theory (eq. [1.1]). Smaller $C_{1}$ means slower migration. These results correspond to surviving protoplanets at $t \sim \tau_{\text {dep }}$ for $\tau_{\text {dep }}=10^{5}, 10^{6}$, and $10^{7}$ years, although depletion of $f_{g}$ on time scales $\tau_{\text {dep }}$ is not taken into account in this result (in the Monte Carlo simulations, the exponential decay is assumed).

The results show that $\Sigma_{d}$ is depleted in an inside-out manner. Ida \& Lin (2008a) found through analytical argument that significant depletion occurs at

$$
r \lesssim a_{\mathrm{dep}, \mathrm{mig}} \simeq C_{1}^{-1 / 8}\left(\frac{f_{g, 0}}{3}\right)^{1 / 40}\left(\frac{t}{10^{6} \mathrm{yrs}}\right)^{1 / 4}\left(\frac{M_{*}}{M_{\odot}}\right)^{3 / 8} \mathrm{AU},
$$

for $C_{1} \gtrsim 0.1$. This boundary is in agreement with the critical location within which $\Sigma_{d}$ has reduced from its initial values by an order of magnitude. Note that the dependences of $a_{\mathrm{dep}, \mathrm{mig}}$ on $C_{1}$ and $f_{g, 0}$ are very weak. This is because for larger $C_{1}$ and/or $f_{g, 0}$, embryos start migration at smaller mass and more number of embryos must be generated to clear the surface density. 

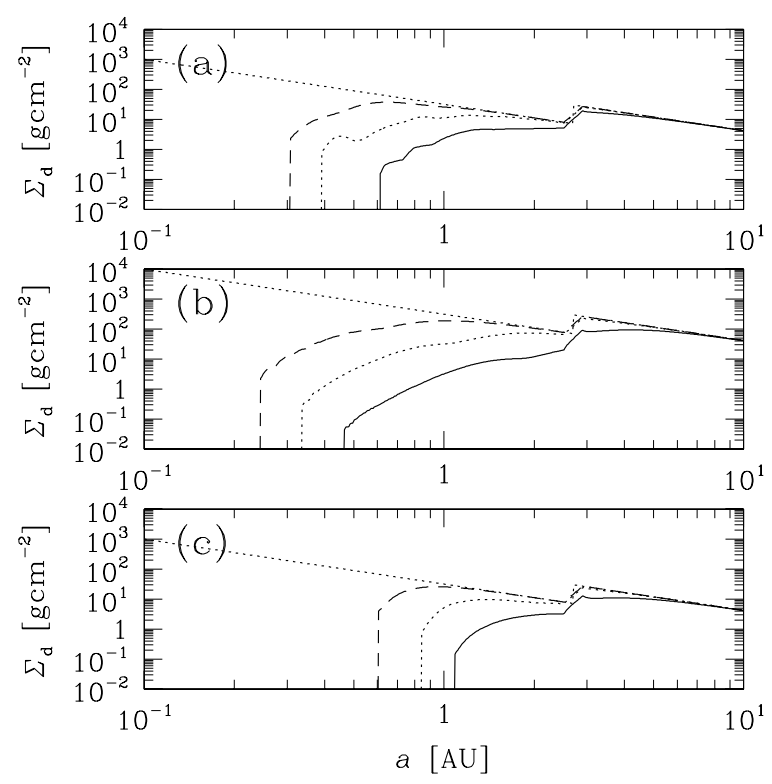

Figure 1. The evolution of $\Sigma_{d}$ due to accretional sculpting by type-I migration for (a) $C_{1}=1$ and $f_{g, 0}=3$, (b) $C_{1}=1$ and $f_{g, 0}=30$, and (c) $C_{1}=0.1$ and $f_{g, 0}=3$. In this plot, we assume constant $f_{g}$. The distributions at $t=10^{5}, 10^{6}$, and $10^{7}$ years are expressed by dashed, dotted, and solid lines. The initial distribution is also shown by dotted lines.

During the early epoch of disk evolution, embryos form and migrate repeatedly to clear out the residual planetesimals. For larger $f_{g, 0}$, the clearing is faster initially. Eventually, the disk gas is so severely depleted that relatively massive embryos no longer undergo significant amount of type-I migration. As shown in Figures 1, this self-regulation process retains the total mass of surviving embryos in terrestrial planet regions that is comparable to or smaller than that of the MMSN model. The typical individual mass of the surviving embryos is $\sim 0.1 M_{\oplus}$. These embryos can coalesce through giant impacts during and after the severe depletion of the disk gas (Kominami \& Ida 2002).

Type-I migration leads to clearing of planetesimals close to their host stars and sets the inner edge of the embryos' population at $\sim 1 \mathrm{AU}$. N-body simulations show that the most massive terrestrial planets tend to form in inner regions of the computational domain where the isolated embryos are initially placed. The lack of planets inside the Mercury's orbit in our Solar system might also be attributed to this result. The selfregulated clearing process ensures the formation of Mars to Earth-sized terrestrial planets in habitable zones. Even in the limit of large $f_{d, 0}$, the reduction of $\Sigma_{d}$ at $\lesssim$ a few AU inhibits in situ formation of gas giants interior to the ice line and facilitates the formation and retention probability of habitable terrestrial planets.

Although the formation of Mars to Earth-sized habitable planets depends only weakly on type-I migration speed, it is critical for the formation of cores of gas giant planets. For giant planets to actually form, sufficiently massive cores that accrete gas on timescales at least shorter than a few folding time of $\tau_{\text {dep }}$ must be formed. For $\tau_{\text {dep }} \sim 10^{6}-10^{7}$ years, we deduce, from eq. (1.2) with $k 1=9$ and $k 2=3$, that gas giant formation is possible only for $M_{\mathrm{c}} \gtrsim$ several times $M_{\oplus}$. But, type-I migration suppresses the emergence of such massive cores in disk regions with relatively large $\Sigma_{g}$. For $C_{1} \gtrsim 0.1$, the asymptotic core masses are generally much smaller than that needed to launch efficient gas accretion even though there is little decline in the magnitude of $\Sigma_{d}$. 


\subsection{Mass - semimajor axis distributions}

In the Monte Carlo simulations, we first generate a 1,000 set of disks with various surface density of gas $\left(\Sigma_{g}\right)$ and planetesimals $\left(\Sigma_{d}\right)$. For each disk, 15 semimajor axes of the protoplanetary seeds are selected from a log uniform distribution in the ranges of $0.05-$ $50 \mathrm{AU}$, assuming that averaged orbital separation between planets is 0.2 in log scale (the averaged ratio of semimajor axes of adjacent planets is $\simeq 1.6$ ). We also assume that $\tau_{\text {dep }}$ and $M_{*}$ have log uniform distributions in the range of $10^{6}-10^{7}$ yrs and $0.8-1.25 M_{\odot}$, respectively. (We focus on the effects of type-I migration but not dependences on stellar mass, so we consider solar-type stars). Corresponding to mm observations of T Tauri disks (e.g., Beckwith \& Sargent 1996), it is assumed that $f_{g, 0}$ has a log normal distribution which is centered on the value of $f_{g, 0}=1$ with a dispersion of $1\left(\delta \log _{10} f_{g, 0}=1.0\right)$ for $M_{*}=M_{\odot}$. We assume $L_{*} \propto M_{*}^{4}$ and the distribution of $f_{g, 0}$ is shifted in proportion to $M_{*}^{2}$ (Ida \& Lin 2005). The choice of the $M_{*}$-dependences do not affect the results, because the range of $M_{*}$ that we consider here is relatively narrow.

Figures $2 \mathrm{a}$ show the predicted $M_{p}-a$ distributions for $C_{1}=0.03,0.1,0.3$ and 1 . We also plot data of extrasolar planets around stars with $M_{*}=0.8-1.25 M_{\odot}$ discovered by radial velocity surveys (http://exoplanet.eu/). The planet masses $M_{p}$ is a factor of $4 / \pi$ times the values of $M_{p} \sin i$ determined from radial velocity measurements, assuming a sample of planetary systems with randomly oriented orbital planes.

Formation probability of gas giants dramatically changes with $C_{1}$. In order to quantitatively compare with observations, we determine the fraction $\left(\eta_{J}\right)$ of stars with planets within the detectability limit by the magnitude of radial velocity $\left(v_{r}>10 \mathrm{~m} / \mathrm{s}\right)$ and orbital periods ( $T_{K}<4$ years). Because we artificially terminate type-I and II migrations near disk inner edge at a 2 day period $\left(\simeq 0.03 \mathrm{AU}\right.$ for $\left.M_{*}=1 M_{\odot}\right)$ and we have not specified a survival criterion for the close-in planets, we exclude close-in planets with $a<0.05 \mathrm{AU}$ in the evaluation of $\eta_{J}$. The values of $\eta_{J}$ are $12.8,3.7,0.4$, and $0 \%$ for the models with $C_{1}=0.03,0.1,0.3$, and 1 , respectively. Only for $C_{1} \lesssim 0.03$, the predicted $\eta_{J}$ can be comparable to the observed data (Fischer \& Valenti 2005). In models with higher $C_{1}$, only the low-mass cores can survive type-I migration. The envelope contraction time scales for these low-mass cores are generally much longer (eq. [1.2]) than the gas depletion time scales. Consequently, $\eta_{J}$ is very small. Ida \& Lin (2008a) showed that a mass function of close-in planets is also sensitively dependent on the magnitude of $C_{1}$ and it will be able to be calibrated from an observed mass function of close-in planets.

In these models, we approximate the gas accretion rate with $(k 1, k 2)=(9,3)$ in eq. (1.2). In view of uncertainties in the gas accretion rate, we also simulated models with $k 1=8$ and 10 , with $k 2=3$ for all cases. For models with $C_{1}=0.03-0.1$ in which type-I migration marginally suppresses the formation of gas giants, the magnitude of $\eta_{J}$ depends sensitively on the minimum mass for the onset of dynamical gas accretion (which is represented by $k 1$ ). A smaller value of $k 1(=8)$ can lead to a significant increase in $\eta_{J}$ because smaller mass cores can initiate the runaway gas accretion within $\tau_{\text {dep }} \sim 10^{6}-10^{7}$ years (eq. [1.2]).

The survival of terrestrial planets depends on their post-formation encounter probability with migrating giant planets. In the absence of any type-I migration, this probability is modest. But the inclusion of a small amount of type-I migration significantly reduces the fraction of stars with massive close-in gas giants because the retention of the progenitor cores becomes possible only at the late stages of disk evolution when the magnitude of $\Sigma_{g}$ is reduced and type-II migration is no more efficient. Repeated migration of gas giants is less common in models with $C_{1} \gtrsim 0.01$ than those with $C_{1}=0$. The low type-II migration probability reduced the need for efficient disruption of largely accumulated 
(a)

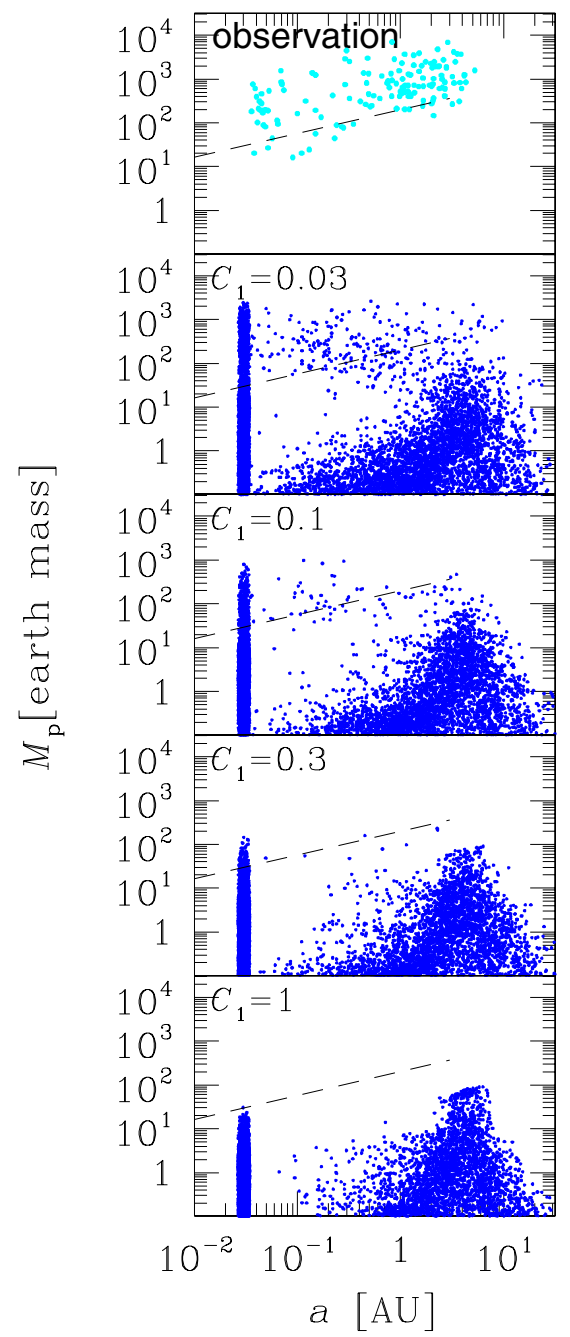

(b)

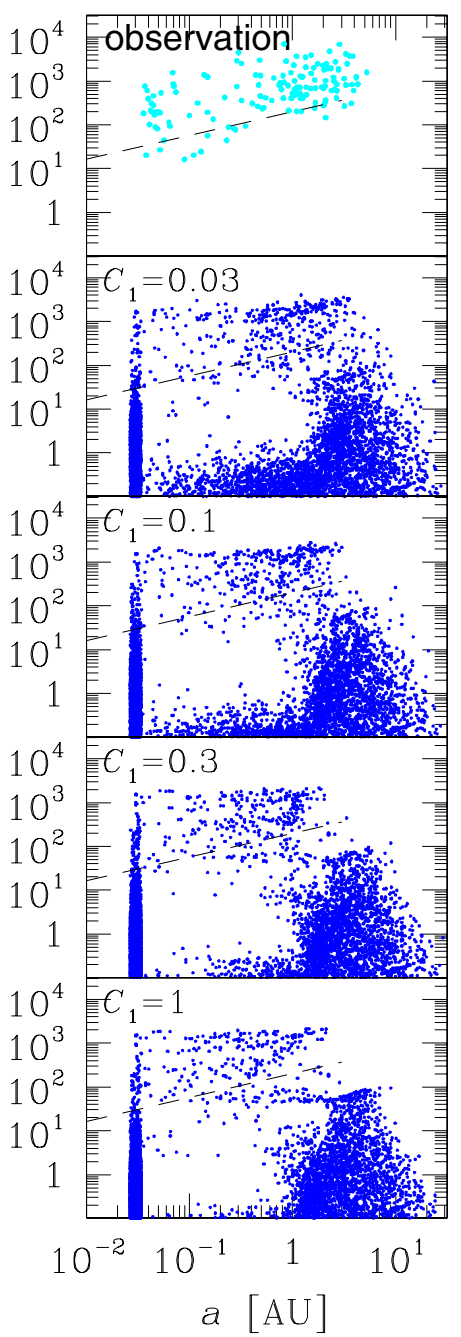

Figure 2. The mass and semimajor axis distribution of extrasolar planets. Units of the mass $\left(M_{\mathrm{p}}\right)$ and semimajor axis $(a)$ are Earth mass $\left(M_{\oplus}\right)$ and AU. (a) Disks with $\Sigma_{g}$ of a power-law radial dependence and (b) Disks with a bump in $\Sigma_{g}$ due to the coupling effect of MRI activity and the ice line. The top panels are observational data of extrasolar planets (based on data in http://exoplanet.eu/) around stars with $M_{*}=0.8-1.25 M_{\odot}$ that were detected by the radial velocity surveys. The determined $M_{p} \sin i$ is multiplied by $1 /\langle\sin i\rangle=4 / \pi \simeq 1.27$, assuming random orientation of planetary orbital planes. The other panels are theoretical predictions with $M_{*}=0.8-1.25 M_{\odot}$ for various values of $C_{1}$. The dashed lines express observational limit with radial-velocity measure precision of $v_{r}=10 \mathrm{~m} / \mathrm{s}$. In these models, the magnitude of the metallicity $[\mathrm{Fe} / \mathrm{H}]=0.1$ and the contraction time scale parameters in eq. (1.2) are assumed to be $(k 1, k 2)=(9,3)$.

close-in planets. It also ensures that most of the terrestrial planets formed in the HZs are not removed by the migrating gas giants. Note that type-I migration also inhibits in situ formation of gas giants near $1 \mathrm{AU}(\S 2.2)$. Thus, a small amount of type-I migration facilitates formation and retention of terrestrial planets in HZs in extrasolar planetary systems, rather than inhibits them. 
Ida \& Lin (2004b) showed through a model without the effects of type-I migration that $\eta_{J}$ increases with $[\mathrm{Fe} / \mathrm{H}]$. The correlation reflects the fact that high $[\mathrm{Fe} / \mathrm{H}]$ enhances formation of cores large enough for runaway gas accretion. The effect of type-I migration enhances the $\eta_{J}-[\mathrm{Fe} / \mathrm{H}]$ correlation, because type-I migration is more efficient in metalpoor disks (Ida \& Lin 2008a). The steep dependence is in a better agreement with the observed data (Fischer \& Valenti 2005).

The dependence on $M_{*}$ was studied by Ida \& Lin (2006), using a simple model without the effects of type-I migration. They predicted that gas giants are much more rare around M dwarfs than around FGK dwarfs while super-Earths are abundant around M dwarfs. These conclusions are not changed by the effects of type-I migration (Ida \& Lin 2008a). Around M dwarfs, super-Earths at 1-3AU, which are inferred to be abundant by microlensing survey, survive type-I migration.

\section{Disks with non-uniform surface density}

\subsection{Disk model}

The surface density of planetesimals is the same as eq. (2.1). However, in order to take into account the effect of spatial non-uniformity of the alpha parameter $(\alpha)$ of disk viscosity (Shakura \& Sunyaev 1973), that of gas is modeled as

$$
\begin{aligned}
& \Sigma_{g}=\frac{\dot{M}}{3 \pi \nu} \\
& \dot{M}=3 \times 10^{-9} f_{g, 0} \exp \left(-t / \tau_{\text {dep }}\right)\left[M_{\odot} / \mathrm{yr}\right]
\end{aligned}
$$

where $\nu=\alpha c_{s} h$. Since the variation of $\Sigma_{g}$ due to change in $\eta_{\text {ice }}$ across $a_{\text {ice }}$ is important, in this section the change in $\eta_{\text {ice }}$ is smoothed out by a tanh function with width of scale height $h$.

Assuming the disk temperature given by eq. (2.2), $\alpha_{\text {active }}=10^{-3}$ and $\alpha_{\text {dead }}=10^{-4}$, and surface density of the surface MRI active layer (Ida \& Lin 2008a),

$$
\Sigma_{\mathrm{A}}=\min \left(6 \eta_{\mathrm{ice}}^{-1}\left(\frac{r}{1 \mathrm{AU}}\right)^{3}\left[\mathrm{~g} / \mathrm{cm}^{2}\right], \Sigma_{g}\right),
$$

the equilibrium $\Sigma_{g}$ distribution is given as a function of $\dot{M}$ in Figure 3. When $\Sigma_{g}$ declines to the values comparable to $\Sigma_{\mathrm{A}}$ near the ice line, the $\Sigma_{g}$ distribution has a positive gradient near the ice line. As mentioned in $\S 1.4$, type-I migration would be halted in the positive gradient regions.

With these prescriptions, we carried out the Monte Carlo simulations to predict mass and semimajor axis of extrasolar planets. The assumed distributions of $f_{g, 0}, \tau_{\mathrm{dep}}$, and $M_{*}$ are the same as those in $\S 2.3$. The mass accretion rate $(\dot{M})$ in this range of $f_{g, 0}$ corresponds to late T Tauri stage (Calvet, Hartmann \& Strom 2000). Figures 2b show the predicted $M_{p}-a$ distributions for $C_{1}=0.03,0.1,0.3$ and 1 . The values of $\eta_{J}$ are $16.2,14.3,11.9$, and $9.5 \%$ for the models with $C_{1}=0.03,0.1,0.3$, and 1 , respectively. Compared with the case without the ice line effect (Figures 2a), $\eta_{J}$ is enhanced, in particular for relatively large $C_{1}$, and the dependence on $C_{1}$ is significantly weakened. Even for $C_{1} \simeq 0.3-1$, the predicted $\eta_{J}$ can be comparable to the observed data. Cores are trapped near the ice line, almost independent of the strength of type-I migration, and they accrete planetesimals until they can start runaway gas accretion.

The active layer thickness given by eq. (3.2) is ten times higher than that used in Kretke \& Lin (2007), because it has large uncertainty and we intended to highlight the importance of the effect of the ice line. Figures 2 suggest that the coupling effect of MRI 


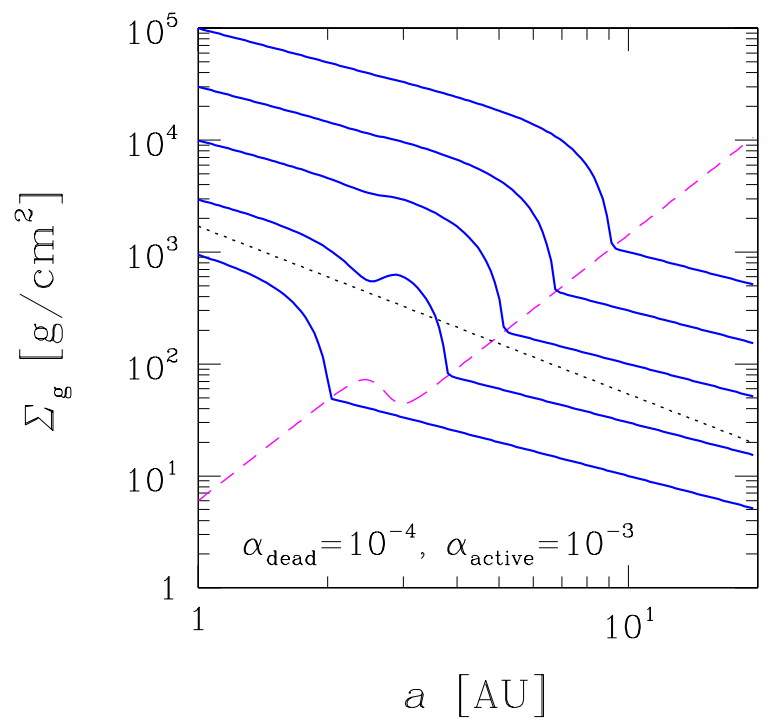

Figure 3. The evolution of $\Sigma_{g}$ with the coupling effect of MRI and the ice line. The solid lines express the $\Sigma_{g}$ distributions for $\dot{M}=10^{-7}, 3 \times 10^{-8}, 10^{-8}, 3 \times 10^{-9}, 10^{-9} M_{\odot} /$ yr from top to bottom. The dashed and dotted lines are $\Sigma_{\mathrm{A}}$ and $\Sigma_{g}$ of the MMSN model.

activity and the ice line can enhance formation and retention rates of gas giants without significant reduction of the strength of type-I migration.

Since the formation and retention of gas giants are facilitated only in the regions beyond the ice line, in situ formation of gas giants in HZs are still inhibited by type-I migration. Figures 2 suggest that the formation probability of hot jupiters is apparently low in the cases with the ice line effect compared with the cases without the effect for similar $\eta_{J}$. Hence, the property that type-I migration facilitates formation and retention of terrestrial planets in HZs is preserved.

The effect of the ice line also preserves the dependence on $M_{*}$ and the correlation between $\eta_{J}$ and $[\mathrm{Fe} / \mathrm{H}]$. But, the correlation is now similar to the case without type-I migration, which is weaker than that in the cases with type-I migration but without the ice line effect. Because the results shown here are limiting cases in which the ice line effect is maximally efficient, realistic cases may be in between the results here and the results without the ice line effect.

\section{Summary}

We have investigated the effects of type-I migration of protoplanetary embryos on mass and semimajor axis distributions of extrasolar planets, through Monte Carlo simulations. In disks with gas surface density having a power-law radial dependence, the type-I migration speed has to be an order of magnitude slower than that given by the linear theory, to be consistent with the existing observational data of extrasolar gas giants. However, a bump in gas surface density can be produced by change in the thickness of MRI active layer near the ice line. The bump can trap migrating protoplanetary embryos. Even with the type-I migration strength similar to that predicted by the linear theory, the formation/retention probability of gas giants can be comparable to the observational data. 
The introduction of type-I migration preserves the dependence on $M_{*}$ that gas giants are much more rare around $M$ dwarfs than around FGK dwarfs while super-Earths are abundant around $M$ dwarfs. The correlation that $\eta_{J}$ increases with $[\mathrm{Fe} / \mathrm{H}]$ is enhanced by the effect of type-I migration. It is in a better agreement with the observed data, although the enhancement is weak in the disk with the bump.

The introduction of type-I migration inhibits in situ formation of gas giants in HZs and reduces the probability of passage of gas giants through HZs, both of which facilitate retention of terrestrial planets in HZs. The strength of type-I migration can be constrained by a mass function of close-in planets, which will be tested by transit observation from space.

\section{References}

Baruteau, C. \& Masset, F. 2008, this proceedings

Beckwith, S. V. W. \& Sargent, A. I., 1996, Nature, 383, 139

Calvet, N., Hartmann, L. \& Strom, S. E. 2000, in Protostars and Planets IV, ed. V. Mannings, A. P. Boss and S. S. Russell (Tucson:Univ. of Arizona Press), 377

Daisaka, K. J., Tanaka, H. \& Ida, S. 2006, Icarus, 185, 492

Davis, S. S. 2005, ApJ, 620, 994

Fischer, D. A. \& Valenti, J. A. 2005, ApJ, 622, 1102

Fromang, S., Terquem, C. \& Nelson, R. P. 2005, MNRAS, 363, 943

Garaud, P. \& Lin, D. N. C. 2007, ApJ, 654, 606

Goldreich, P., \& Tremaine, S. 1980, ApJ, 241, 425

Guillot, T. \& Hueso, R. 2006, MNRAS, 367, L47

Hayashi, C. 1981, Prog. Theor. Phys. Suppl., 70, 35

Ida, S. \& Lin, D. N. C. 2004a, ApJ, 604, 388

Ida, S. \& Lin, D. N. C. 2004b, ApJ, 616, 567

Ida, S. \& Lin, D. N. C. 2005, ApJ, 626, 1045

Ida, S. \& Lin, D. N. C. 2008, ApJ, in press

Ida, S. \& Lin, D. N. C. 2008, in preparation

Kokubo, E. \& Ida, S. 1998, Icarus, 131, 171

Kokubo, E. \& Ida, S. 2002, ApJ, 581, 666

Kominami, J. \& Ida, S. 2002, Icarus, 157, 43

Koller, J., Li, H. 2004, in The Search For Other Worlds. AIP Conf. Proc., 713, pp. 63-66

Kretke, K. A. \& Lin, D. N. C. 2007, ApJ, 664, L55

Laughlin, G., Steinacker, A. \& Adams, F. C. 2004, ApJ, 309, 846

Li, H. et al. 2005, ApJ, 624, 1003

McNeil, D., Duncan, M. \& Levison, H. F. 2005, AJ, 130, 2884

Marcy, G. et al. 2005, Prog. Theor. Phys. Suppl., 158, 24

Masset, F. S., D'Angelo, G. \& Kley, W., 2006, ApJ, 652, 730

Masset, F. S., Morbidelli, A., Crida, A., Ferreira, J., 2006, ApJ, 642, 478

Mayor, M., Pont, F. \& Vidal-Madjar, A. 2005, Prog. Theor. Phys. Suppl., 158, 43

Muto, T. \& Inutsuka, S. 2008, this proceedings

Nelson, R. P. \& Papaloizou, J. C. B. 2004, MNRAS, 350, 849

Sano, T., Miyama, S. M., Umebayashi, T., \& Nakano, T., 2000, ApJ, 543, 486

Shakura, N. I. \& Sunyaev, R. A. 1973, A\&A, 24, 337

Tanaka, H., Takeuchi, T. \& Ward, W. 2002, ApJ, 565, 1257

Thommes, E. \& Murray, N. 2006, ApJ, 644, 1214

Ward, W. 1986, Icarus, 67, 164

Zhang, X., Lin, D. N. C. \& Kretke, K. A. 2008,in: Y.-S. Sun, S. Ferraz-Mello \& J.-L. Zhou, (eds.), Exoplanets: Detection, Formation and Dynamics, Proc. IAU Symposium No. 249 (Suzhou,China), p. 309 